\title{
A simplified version of TI-RADS exhibits comparable diagnostic performance in malignancy risk stratification of thyroid nodules
}

\section{Qingfang Chen}

The First Affiliated Hospital of Hainan Medical University

SizeWu ( $\sim$ wsz074@aliyun.com )

The First Affiliated Hospital of Hainan Medical University https://orcid.org/0000-0002-1086-764X

\section{Research Article}

Keywords: thyroid nodule; malignancy; ultrasound; thyroid imaging reporting and data system (TIRADS); C-TIRADS; ACR-TIRADS

Posted Date: March 9th, 2021

DOI: https://doi.org/10.21203/rs.3.rs-306585/v1

License: (c) (i) This work is licensed under a Creative Commons Attribution 4.0 International License. Read Full License 


\section{Abstract}

Evaluation of thyroid focal lesions using thyroid imaging reporting and data system (TIRADS) has been proceeding for a decade, but there is no consensus on any version of TIRADS. The purpose of this study was to validate a recently launched simplified Chinese version of TIRADS, with a compare to the American College of Radiology version of TIRADS. A total of 1306 patients with 1389 thyroid nodules were reviewed and assessed according the two TIRADSs, and the histopathological results were taken as golden standard. The results showed there were 973 benign nodules and 416 malignant nodules. The highest accuracies(AUCs) of C-TIRADS 4C and ACR-TIRADS 5 were taken as the optimized cut-off values for diagnosis. The sensitivity, specificity, PPV, NPV and AUC by C-TIRADS 4 C and ACR-TIRADS 5 for thyroid nodule evaluation were $87.39 \%, 89.92 \%, 75.00 \%, 95.38 \%$ and 0.89 , and $85.58 \%, 91.88 \%, 81.84 \%$, $93.71 \%$ and 0.89 , respectively, $(P>0.05$ for all). We concluded that C-TIRADS and ACT-TIRADS have very good diagnostic performance in differentiating malignant from benign thyroid nodules by each optimized cut-off value, and the diagnostic performance of the fewer parameters based C-TIRADS 4C is comparable to the multiple parameters based ACR-TIRADS 5.

\section{Introduction}

Thyroid nodular lesions are common in adult population. The prevalence of thyroid nodules in adults is about 19-68\% (Haugen et al.2016; Jiang et al.2016; Kitahara et al.2016; Morris et al.2016; Moon et al.2018), and that of malignant lesions is about 6.7-15\% (Haugen et al.2016; Kitahara et al.2016; Morris et al.2016). Thyroid nodules comprise of benign, borderline and malignant lesions, and benign nodules are much more common than malignant nodules (Haugen et al.2016). Timely detection and accurate diagnosis of benign and malignant thyroid nodules are of significance for clinical management. However, malignant nodules before distal metastasis usually have no unique clinical manifestations and laboratory abnormalities, which makes it difficult to differentiate malignant nodule from benign nodule (Haugen et al.2016; Kwak et al.2011; Russ et al.2017; Shin et al.2016; Tessler et al.2017; Zhou et al.2020). High-resolution color Doppler ultrasound is the mostly used imaging modality for the evaluation of thyroid nodules and has some advantages, but the diagnostic performance is not satisfactory (Kwak et al.2011; Russ et al.2017; Shin et al.2016; Tessler et al.2017; Zhou et al.2020). In view of this disadvantage, clinical researchers have proposed a series of thyroid nodule risk stratification systems for thyroid nodules through research on the ultrasound features of thyroid nodules, in order to improve the diagnosis and treatment of thyroid nodules. Starting with the establishment of the Thyroid Imaging Reporting and Data System (TIRADS) in 2009 by Horvath et al, different versions of TI-RADS have been proposed (Horvath et al.2009). However, due to the similarities and discordance in terms and standards in the description and definitions of the ultrasound features of thyroid nodules adopted by different researchers when establishing the classification systems, and no system has been widely acknowledged and used so far (Fradin et al.2020; Grani et al.2019; Ha et al.2018, 2019; Horvath et al.2009; Kwak et al.2011; Russ et al.2017; Shin et al.2016; Tessler et al.2017; Xu et al.2019; Yoon et al.2019; Zhou et al.2020). There are some discordance in the number of evaluation parameters among different versions 
of TIRADS, such as ACR-TIRADS by the American College of Radiology, 23 parameters are used for scoring; and the recently launched Chinese version TIRADS (C-TIRADS) by The Superficial Organ and Vascular Ultrasound Group of the Society of Ultrasound in Medicine of Chinese Medical Association, 6 parameters are used for scoring (Zhou et al.2020). Multi-parameter scoring has the advantage of being systematic and comprehensive, but it is not convenient for application. If fewer parameters can work effectively, it will be better. Whether simplified fewer parameters can compromise or increase risk stratification level and diagnostic performance has not been investigated. The objective of this study was to compare the diagnostic performances of TIRADSs based on multiple and fewer parameters and validate the C-TIRADS.

\section{Materials And Methods Study population}

Patients who had undergone thyroid ultrasound examination and core needle biopsy or surgery in the First Affiliated Hospital of Hainan Medical University from January 2015 to December 2020 were selected as the potential research subjects, and the relevant data were retrospectively analyzed. Inclusion criteria: Patients with eligible quality sonographic images of thyroid nodule and had undergone histopathological study. Exclusion criteria: The ultrasound images of thyroid nodule were of poor quality or the image number was not enough, which could not fully display the nodular features; and an indeterminate histopathology. A single conglomerate of nodules was counted as one nodule. If a patient with several thyroid nodules, the highly suspicious malignant nodules and one of representative benign nodules were enrolled. A total of 1306 patients with 1389 thyroid nodules were included, and 42 patients with 58 thyroid nodules were excluded. Histopathological results of thyroid nodules were used as reference standard to determine the benign and malignant nature of nodules. The histopathological diagnosis was made according to the diagnostic criteria of the World Health Organization (Cameselle-Teijeiro et al.2018). Thyroid ultrasound imaging had been performed before surgery in all patients with thyroid nodular lesions. The C-TIRADS and the ACR-TIRADS were identified by retrospective analysis of ultrasound images by a physician with 16 years of experience in thyroid ultrasound evaluation.

This study was approved by the Institutional Review Board of the First Affiliated Hospital of Hainan Medical University, and informed consent was waived due to the retrospective design.

\section{Ultrasound examination}

Multi-parameter ultrasound systems (Siemens Acuson S2000; Mindray DC 8; Mindray Resona 7区 Aloka Prosound a-7区 Aloka Prosound a-10; Phillips EPIQ5 and GE Logiq E9) were used for thyroid examination. During the examination, the ultrasound systems were adjusted to small parts mode (thyroid gland), and a linear array transducer with a frequency of $5-14 \mathrm{MHz}$ was used. During the examination, the patient took a supine position with head retroversion to fully expose the neck. The thyroid was scanned by cross and longitudinal sections to detect any lesions. If a thyroid nodule was found, its location, shape, orientation(ratio of anterior diameter to transverse diameter), margin(capsule), boundary, size, 
architecture(composition) and internal echogenicity, posterior acoustic effect, vascularity, and relation to abut tissue were identified and scrutinized. Absence or presence of abnormal lymph node in the neck was noted, with attention to the presence of punctuate hyperechoic foci and calcification. Representative images were saved in Picture Archiving and Communications Systems (PACS).

Assessment of thyroid nodules with reference to C-TIRADS (Zhou et al.2020).

Assessing a thyroid and thyroid nodule with C-TIRADS involves a comprehensive evaluation of its composition(architecture), echogenicity, shape (orientation), margin, and echogenic foci. Score points are assigned to optimized sonographic feature, with higher values indicating greater degrees of suspicion. The total score point of a nodule is calculated for classification to assign its TIRADS category, comprising seven categories from no nodule to benign, and highly suspicious malignancy and histology confirmed malignant nodule. Nodule-free thyroid was categorized as TIRADS 1, nodule with - 1 score point was categorized as TIRADS 2, nodule with 0 score point was categorized as TIRADS 3 , nodule with 1 score point was categorized as TIRADS 4A, nodule with 2 score points was categorized as TIRADS 4B, nodule with 3 or 4 score points was categorized as TIRADS 4C, nodule with 5 score point was categorized as TIRADS 5, nodule with histopathology confirmed malignancy was categorized as TIRADS 6;and entirely simple cystic nodule or spongy nodule was categorized as TIRADS 2 . Sonographic feature for score point: Solid composition $=+1$, markedly hypoechoic $=+1$, taller than wide $(A / W \geq 1)=+1$, illdefined or irregular or lobulated or extrathyroidal extension $=+1$, hyperechogenicity with comet-tail artifact $=-1$, punctate hyperechogenicity (suspicious calcification) $=+1$; if there are several hyperechoic patterns in a nodule, only the highest scored is included, and the others are excluded.

Assessment of thyroid nodules with reference to ACR TI-RADS (2017) (Tessler et al.2017).

Assessing a nodule with ACR-TIRADS involves a comprehensive evaluation of its composition, echogenicity, shape (orientation), margin, and echogenic foci. Score points were assigned to each sonographic feature, with higher values indicating greater degrees of suspicious malignancy. The total score of a nodule was calculated for classification to assign its TIRADS category, comprising five categories from benign to highly suspicious malignancy. The total score point of 0 was categorized as TIRADS 1, 2 as TIRADS 2, 3 as TIRADS 3, 4-6 as TIRADS 4, and 7 points or more as TIRADS 5 . Nodular composition (architecture) for score: Cystic $=0$, spongy $=0$, mixed solid-cystic with dominant cystic $=1$, solid $=2$, dominant solid or indeterminate $=2$. Internal echogenicity for score: Anechoic $=0$, isoechoic $=1$, hyperechoic $=1$, indeterminate $=1$, hypoechoic $=2$, markedly hypoechoic $=3$. Shape (orientation) for score: 0 point for wider than tall $(A / W<1)$, and 3 points for taller than wide $(A / W \geq 1)$. Margin for score point: Smooth $=0$, ill-defined $=0$, irregular $=2$, lobulated $=2$, extrathyroidal extension $=3$. Echogenic foci for score point: None $=0$, hyperechoic with comet-tail artifact $=0$, macrocalcifications $=1$, peripheral or rim hyperechoic $=2$, punctate hyperechoic $=3$. Figures $1-3$ illustrate some sonographic features of thyroid nodules and scoring of TIRADS.

\section{Statistical analysis}


Quantitative data with normal distribution was expressed as mean and standard deviation ( $M \pm S D)$, quantitative data do not show normal distribution and qualitative data were expressed as median (interquartile range, IQR) and percentile. The consistency between C-TIRADS and ACR-TIRADS was studied, and the malignant risk stratification levels corresponding to each TIRADS were evaluated. Paired samples t-test and independent samples $T$ test were used to test quantitative data with normal distribution. Qualitative data were analyzed by nonparametric test or Chi-square test. The receiver operating characteristic (ROC) curve area under curve (AUC) was used to evaluate the diagnostic performance of the two TIRADS, and the sensitivity, specificity, positive predictive value (PPV), and negative predictive value (NPV) were calculated. The highest accuracy was taken as the best threshold for diagnosis. All statistical analyses were performed using SPSS statistical software version 23 (IBM Corp., Armonk, NY, USA) and Medcalc statistical software version 15.2.2 (Medcalc software BVBA, Ostend, Belgium), and a $P<0.05$ was considered significant difference.

\section{Results}

Of the 1306 patients with 1389 thyroid nodules, there were 973 benign nodules and 416 malignant nodules, and demographic and ultrasound features of the patients with thyroid nodules are summarized in Table 1. Papillary carcinomas accounted for $96.88 \%$ of malignant nodules, nodular goiter accounted for $93.32 \%$ of benign nodules, and details of the distribution of pathologies of thyroid nodules are listed in Table 2. The highest accuracies(AUCs) of C-TIRADS 4C and ACR-TIRADS 5 were taken as the optimized cut-off values (thresholds) for diagnosis. Taking optimized category of C-TIRADS 4C as cut-off, 312 nodules were confirmed true positive (malignant lesion), 104 nodules were confirmed false positive (benign lesion), 928 nodules were confirmed true negative (benign lesion), and 45 nodules were confirmed false negative (malignant lesion). Taking optimized category of ACR-TIRADS 5 as cut-off, 356 nodules were confirmed true positive (malignant lesion), 79 nodules were confirmed false positive (benign lesion), 894 nodules were confirmed true negative (benign lesion), and 60 nodules were confirmed false negative (malignant lesion). The sensitivity, specificity, PPV, NPV and AUC by C-TIRADS 4C and ACR-TIRADS 5 for thyroid nodule evaluation were $87.39 \%, 89.92 \%, 75.00 \%, 95.38 \%$ and 0.89 , and $85.58 \%, 91.88 \%, 81.84 \%, 93.71 \%$ and 0.89 , respectively, and there was no significant difference between them ( $P>0.05$ for all), as summarized in Table 3. There were 112 benign nodules and 193 malignant nodules with maximal diameter $<10 \mathrm{~mm}$; comparison of diagnostic performance between two TI-RADSs for nodules with different sizes showed that there was no significant difference between nodules with maximal diameter $<10 \mathrm{~mm}$ and $\geq 10 \mathrm{~mm}$ by the cut-off of C-TIRADS $4 \mathrm{C}$ and ACR-TIRADS 5 for the sensitivity, specificity, PPV, NPV ( $P>0.05$ for all), and the AUC of C-TIRADS 4C was significantly lower than that of ACR-TIRADS 5 for thyroid nodules with maximal diameter $<10 \mathrm{~mm}(P=0.0015)$, and the sensitivity and specificity had no significant difference, as summarized in Table 4. The AUC of C-TIRADS $4 \mathrm{C}$ for the nodules with maximal diameter $<10 \mathrm{~mm}$ was significantly lower than the AUC of C-TIRADS 4C for all size nodules $(P<0.001)$. 
Table 1

Demographic and ultrasound features of the patients with thyroid nodules

\begin{tabular}{|c|c|c|c|}
\hline Characteristics & $\begin{array}{l}\text { Benign nodules }(n= \\
973)\end{array}$ & $\begin{array}{l}\text { Malignant nodules }(\mathrm{n}= \\
416)\end{array}$ & $P$ \\
\hline \multicolumn{4}{|l|}{ Age(year) } \\
\hline Mean & $46.09 \pm 12.66$ & $44.69 \pm 12.59$ & 0.059 \\
\hline Range & $7-82$ & $7-84$ & \\
\hline \multicolumn{4}{|l|}{ Gender } \\
\hline Male(n,\%) & $222(22.82)$ & $102(24.52)$ & 0.489 \\
\hline Female(n,\%) & $751(77.18)$ & $314(75.48)$ & \\
\hline \multicolumn{4}{|l|}{ Size(mm) } \\
\hline Mean & $23.02 \pm 10.93$ & $12.58 \pm 8.57$ & $<.001$ \\
\hline Range & $(2-65)$ & $(2-67)$ & \\
\hline$<10 \mathrm{~mm}(\mathrm{n}, \%)$ & $112(11.51)$ & 193(46.39) & $\begin{array}{l}< \\
0.001\end{array}$ \\
\hline$\geq 10 \mathrm{~mm}(\mathrm{n}, \%)$ & $861(88.49)$ & $223(53.61)$ & \\
\hline \multicolumn{4}{|l|}{ Number } \\
\hline Single(n,\%) & $61(6.27)$ & $58(13.94)$ & $<.001$ \\
\hline Multiple(n,\%) & 912(93.73) & $358(86.06)$ & \\
\hline \multicolumn{4}{|l|}{ Composition } \\
\hline Cystic/spongiform(n,\%) & $55(5.65)$ & $0(0.00)$ & $<.001$ \\
\hline $\begin{array}{l}\text { Mixed cystic } \\
\text { and solid(n,\%) }\end{array}$ & $457(46.97)$ & $12(2.88)$ & $<.001$ \\
\hline Solid(n,\%) & $461(47.38)$ & 404(97.12) & $<.001$ \\
\hline \multicolumn{4}{|l|}{ Echogenicity } \\
\hline Anechoic(n,\%) & $33(3.40)$ & $0(0.00)$ & <. \\
\hline Iso/hyperechoic(n,\%) & $540(55.49)$ & $41(9.86)$ & $\begin{array}{l}< \\
0.001\end{array}$ \\
\hline
\end{tabular}




\begin{tabular}{|c|c|c|c|}
\hline Characteristics & $\begin{array}{l}\text { Benign nodules }(n= \\
973)\end{array}$ & $\begin{array}{l}\text { Malignant nodules }(n= \\
\text { 416) }\end{array}$ & $P$ \\
\hline Hypoechoic(n,\%) & $382(39.26)$ & $306(73.56)$ & $\begin{array}{l}< \\
0.001\end{array}$ \\
\hline Marked hypoechoic(n,\%) & $18(1.85)$ & $69(16.58)$ & $\begin{array}{l}< \\
0.001\end{array}$ \\
\hline \multicolumn{4}{|l|}{ Shape } \\
\hline Wider-than-tall(n,\%) & $938(96.40)$ & 233(56.01) & $<.001$ \\
\hline Taller-than-wide(n,\%) & $35(3.60)$ & 183(43.99) & \\
\hline \multicolumn{4}{|l|}{ Margin } \\
\hline Smooth/ill-defined(n,\%) & $917(94.24)$ & 158(37.98) & $\begin{array}{l}<.001 \\
0.001\end{array}$ \\
\hline Lobulated/irregular(n,\%) & $54(5.55)$ & $219(52.64)$ & $\begin{array}{l}< \\
0.001\end{array}$ \\
\hline Extrathyroid extension(n,\%) & $2(0.21)$ & $39(9.38)$ & $\begin{array}{l}< \\
0.001\end{array}$ \\
\hline \multicolumn{4}{|l|}{ Echogenic foci } \\
\hline $\begin{array}{l}\text { None or large comet-tail } \\
\text { artifacts }(n, \%)\end{array}$ & $809(81.47)$ & $88(21.15)$ & $<.001$ \\
\hline Macrocalcifications(n,\%) & $73(7.35)$ & $28(6.73)$ & 0.653 \\
\hline Peripheral calcifications(n,\%) & $18(1.81)$ & $7(1.68)$ & 1.000 \\
\hline Punctate echogenic foci(n,\%) & 93(9.37) & 293(70.44) & $\begin{array}{l}<.001 \\
0.001\end{array}$ \\
\hline
\end{tabular}

Table 2

Distribution of pathologies of thyroid nodules

\begin{tabular}{|llll|}
\hline Pathology of benign nodule & Number (\%) & Pathology of malignant nodule & Number (\%) \\
\hline Nodular goiter & $908(93.32)$ & Papillary carcinoma & $403(96.88)$ \\
\hline Follicular adenoma & $35(3.60)$ & Medullary carcinoma & $8(1.92)$ \\
\hline Eosinophilic adenoma & $11(1.13)$ & Follicular carcinoma & $4(0.96)$ \\
\hline Chronic lymphocytic thyroiditis & $16(1.64)$ & Anaplastic carcinoma & $1(0.24)$ \\
\hline Toxic nodular goiter & $1(0.10)$ & & \\
\hline Granulomatous thyroiditis & $2(0.21)$ & & $416(100.00 \%)$ \\
\hline Total & $973(100.00 \%)$ & & \\
\hline
\end{tabular}


Table 3

Diagnostic performances by two TI-RADSs by different cut-off categories

\begin{tabular}{|c|c|c|c|c|c|}
\hline $\begin{array}{l}\text { Cut-off } \\
\text { category }\end{array}$ & Sensitivity(\%) & Specificity(\%) & PPV(\%) & NPV(\%) & AUC \\
\hline $\begin{array}{l}\text { C-TIRADS } \\
4 \mathrm{~B}, 4 \mathrm{C} \text { and } 5\end{array}$ & $\begin{array}{l}71.75(67.71- \\
75.54)\end{array}$ & $\begin{array}{l}95.92(94.37- \\
97.14)\end{array}$ & $\begin{array}{l}91.59(88.49- \\
94.07)\end{array}$ & $\begin{array}{l}84.58(82.16- \\
86.80)\end{array}$ & $\begin{array}{l}0.84(0.82- \\
0.86)\end{array}$ \\
\hline $\begin{array}{l}{ }^{*} \text { C-TIRADS } 4 C \\
\text { and } 5\end{array}$ & $\begin{array}{l}87.39(83.50- \\
90.66)\end{array}$ & $\begin{array}{l}89.92(87.92- \\
91.69)\end{array}$ & $\begin{array}{l}75.00(70.55- \\
79.09)\end{array}$ & $\begin{array}{l}95.38(93.86- \\
96.61)\end{array}$ & $\begin{array}{l}0.89(0.87- \\
0.90)\end{array}$ \\
\hline & $\begin{array}{l}90.32(74.25- \\
97.9)\end{array}$ & $\begin{array}{l}71.43(68.94- \\
73.8)\end{array}$ & & $\begin{array}{l}99.69(99.10- \\
99.94)\end{array}$ & $\begin{array}{l}0.81(0.79- \\
0.83)\end{array}$ \\
\hline \multirow{2}{*}{$\begin{array}{l}\text { ACR-TIRADS } 4 \\
\text { and } 5\end{array}$} & & & \multicolumn{3}{|l|}{$(4.52-9.58)$} \\
\hline & $\begin{array}{l}55.57(51.87- \\
59 .)\end{array}$ & $\begin{array}{l}98.19(96.86- \\
99.0)\end{array}$ & $\begin{array}{l}97.12(95.02- \\
98.50)\end{array}$ & $\begin{array}{l}66.80(63.75- \\
66.76)\end{array}$ & $\begin{array}{l}0.77(0.75- \\
0.79)\end{array}$ \\
\hline \#ACR-TIRADS 5 & $\begin{array}{l}85.58(81.83- \\
88.81)\end{array}$ & $\begin{array}{l}91.88(89.98- \\
93.52)\end{array}$ & $\begin{array}{l}\text { 81.84(77.89- } \\
85.35)\end{array}$ & $\begin{array}{l}93.71(91.98- \\
95.17)\end{array}$ & $\begin{array}{l}0.89(0.78- \\
0.90)\end{array}$ \\
\hline${ }^{* \# P}$ value & 0.7863 & 0.6375 & 0.1421 & 0.8795 & 1.000 \\
\hline \multicolumn{6}{|c|}{$\begin{array}{l}\text { C-TIRADS: The Superficial Organ and Vascular Ultrasound Group of the Society of Ultrasound in } \\
\text { Medicine of Chinese Medical Association version of thyroid imaging, reporting and data system; ACR- } \\
\text { TIRADS: American College of Radiology version of thyroid imaging, reporting and data system;PPV: } \\
\text { Positive predictive value; NPV: Negative predictive value; AUC: Area under the ROC curve; Variables in } \\
\text { paratheses are } 95 \% \text { confidential intervals. }\end{array}$} \\
\hline
\end{tabular}


Table 4

Comparison of diagnostic performance between two TI-RADSs for nodules with different sizes

\begin{tabular}{|c|c|c|c|c|c|}
\hline Characteristics & Sensitivity(\%) & Specificity(\%) & PPV(\%) & NPV(\%) & AUC(\%) \\
\hline \multicolumn{6}{|l|}{$\begin{array}{l}\text { Nodular diameter } \\
<10 \mathrm{~mm}\end{array}$} \\
\hline C-TIRADS & $\begin{array}{l}86.22(81.02- \\
90.44)\end{array}$ & $\begin{array}{l}62.79(53.84- \\
71.14)\end{array}$ & $\begin{array}{l}80.17(74.58- \\
85.00)\end{array}$ & $\begin{array}{l}72.32(63.07- \\
80.36)\end{array}$ & $\begin{array}{l}0.75(0.70- \\
0.79)\end{array}$ \\
\hline ACR-TIRADS & $\begin{array}{l}84.46(78.56- \\
89.26)\end{array}$ & $\begin{array}{l}\text { 83.04(74.78- } \\
89.47)\end{array}$ & $\begin{array}{l}89.56(84.18- \\
93.60)\end{array}$ & $\begin{array}{l}75.61(67.05- \\
82.90)\end{array}$ & $\begin{array}{l}0.84(0.79- \\
0.88)\end{array}$ \\
\hline$P$ value & 0.9424 & 0.1653 & 0.4697 & 0.8417 & 0.0015 \\
\hline \multicolumn{6}{|l|}{$\begin{array}{l}\text { Nodular diameter } \\
\geq 10 \mathrm{~mm}\end{array}$} \\
\hline C-TIRADS & $\begin{array}{l}90.76(85.62- \\
94.53)\end{array}$ & $\begin{array}{l}93.94(92.19- \\
95.40)\end{array}$ & $\begin{array}{l}75.23(69.01- \\
80.76)\end{array}$ & $\begin{array}{l}98.05(96.89- \\
98.86)\end{array}$ & $\begin{array}{l}0.92(0.91- \\
0.94)\end{array}$ \\
\hline ACR-TIRADS & $\begin{array}{l}86.55(81.36- \\
90.74)\end{array}$ & $\begin{array}{l}93.03(91.12- \\
94.64)\end{array}$ & $\begin{array}{l}76.28(70.55- \\
81.39)\end{array}$ & $\begin{array}{l}96.39(94.89- \\
97.55)\end{array}$ & $\begin{array}{l}0.90(0.88- \\
0.92)\end{array}$ \\
\hline$P$ value & 0.8857 & 0.8912 & 0.9442 & 0.8090 & 0.1573 \\
\hline \multicolumn{6}{|c|}{$\begin{array}{l}\text { C-TIRADS: The Superficial Organ and Vascular Ultrasound Group of the Society of Ultrasound in } \\
\text { Medicine of Chinese Medical Association version of thyroid imaging, reporting and data system; ACR- } \\
\text { TIRADS: American College of Radiology version of thyroid imaging, reporting and data system;PPV: } \\
\text { Positive predictive value; NPV: Negative predictive value; } 95 \% \text { Cl: } 95 \% \text { confidential interval. }\end{array}$} \\
\hline
\end{tabular}

\section{Discussion}

The treatment of focal thyroid nodules mainly depends on the benign and malignant pathology. If benign thyroid nodules do not cause compression symptoms and do not impair the cosmetic appearance, the usual management is follow-up. Surgical treatment has been a necessary approach for malignant nodules (Haugen et al.2016). Color Doppler ultrasound has high value in identifying thyroid nodules. However, due to the complex and diverse sonographic features of benign and malignant thyroid nodules, there is partial overlap between them, which makes it difficult to determine the pathology of thyroid nodules without typical features (Haugen et al.2016; Tessler et al.2017). Using scores of different sonographic features of thyroid nodule for risk stratification is an evaluation strategy proposed in recent decades. The results of this study showed that the recently launched simplified version C-TIRADS has high clinical significance for malignancy risk stratification of thyroid nodules, and the AUC with cut-off of C-TIRADS 4C is 0.89, which is equal to that of ACR-TIRADS 5 and higher than that 0.84 for C-TIRADS 4B, $4 C$ and 5 , and 0.81 for C-TIRADS 5 . With C-TIRADS $4 \mathrm{C}$ as the diagnostic threshold, the sensitivity and specificity in the diagnosis of thyroid malignant nodules were $87.39 \%$ and $89.92 \%$, respectively, which are similar to that of ACR-TIRADS 5. AUC of C-TIRADS 5 in our study is lower than the AUCs of C-TIRADS 5 and ACR-TIRADS 5 in the study by Zhou et al, and the AUC of our C-TIRADS 4C is higher than the AUC of ACR-TIRADS 5 in the study by Zhou et al (2020). AUC of ACR-TIRADS 5 in this study is 0.89 , which is 
higher than those in the previous studies of accuracy of 0.52 and AUC of 0.835 and 0.864 (Hoang et al.2018; Magri et al.2020; Peng et al.2020).

There were 112 benign nodules and 193 malignant nodules with maximal diameter $<10 \mathrm{~mm}$ in this study; the AUC of C-TIRADS $4 C$ for the small size nodules was significantly lower than the average AUC of CTIRADS 4C for all size nodules; the AUC of C-TIRADS $4 \mathrm{C}$ was significantly lower than that of ACR-TIRADS 5 for them, and the sensitivity and specificity had no significant difference, indicating that C-TIRADS does not exhibit significant diagnostic efficiency for thyroid nodules with maximal diameter $<10 \mathrm{~mm}$. The AUC of C-TIRADS $4 \mathrm{C}$ for thyroid nodules with maximal diameter $\geq 10 \mathrm{~mm}$ was slightly higher than that of ACRTIRADS 5, but there is no significant difference. The AUC of 0.90 of ACR-TIRADS 5 for thyroid nodules with maximal diameter $\geq 10 \mathrm{~mm}$ is higher than the accuracy of 0.69 reported by $\mathrm{Ha}$ et al (2018).

Similar to ACR-TIRADS, C-TIRADS also evaluates thyroid nodules from five aspects: structural composition, internal echogenicity, shape, margin and boundary, and hyperechoic foci, but the C-TIRADS has more advantages. Firstly, a category for normal thyroid without nodule and a category for histopathology confirmed nodule have been added to C-TIRADS, enabling appropriateness and completeness for the thyroid evaluation. Secondly, the sonographic features for malignant risk stratification adopted by C-TIRADS are more rational, for these chosen features have been optimized and selected through a multiple logistic model with forward stepwise selection and logistic regression analysis, instead of assigning scores to every sonographic feature (Zhou et al.2020). Thirdly, both TIRADS have elaborated, defined and classified the sonographic features of thyroid nodules in detail, and scored according to the sonographic features, but the scoring methods and assigning methods are different (Tessler et al.2017; Zhou et al.2020). For example, the score of fine punctate calcification is 3 points in ACR-TIRADS and 1 point in C-TIRADS; the score of hyperechoic foci with comet tail artifact is 0 point in ACR-TIRADS, and - 1 point in C-TIRADS. The score of ACR-TIRADS involves 23 sonographic features, while C-TIRADS involves only 6 sonographic features, the latter is simplified and clearer. Fourthly, C-TIRADS can reduce radiologist's time for accumulative scoring, and has better clinical practicability. Simplified scoring parameters C-TIRADS can reduce or avoid inaccurate scoring deviation caused by the difference of the scorers' understanding of images, such as treating hypoechoic as markedly hypoechoic, and spongy nodules as cystic-solid mixed nodules. Punctate echogenic foci are easily confused with small comet-tail artifact, and the scoring difference between the two systems is great, which may cause bias and error (Tessler et al.2017; Zhou et al.2020a, 2020b). Fifthly, the risk stratification of C-TIRADS targets directly to the degree of malignancy, and it's convenient for understanding by other physicians.

The threshold interval of C-TIRADS for malignant risk range is appropriate, and the malignant risk of five categories is not less than $90 \%$, and our study results of AUC of 0.89 of C-TIRADS 4C and AUC of 0.81 of C-TIRADS 5 showed high consistency and limited deviation (Zhou et al.2020). The main objective of ACRTIRADS category was to determine recommendations for FNA and follow-up, with a low threshold of malignant risk range from the estimated at $2 \%$ or less for ACR-TIRADS 1 to the greater than $20 \%$ for ACRTIRADS 5, but in this study, the AUC of 0.89 was high, which indicate that many thyroid nodules with ACR- 
TIRADS 5 has much higher malignant risk, other than $20 \%$ or plus, as reported by other studies (Ha et al.2018; Hoang et al.2018; Magri et al.2020; Peng et al.2020; Zhou et al.2020).

In our study, the best threshold for ACR-TIRADS to diagnose thyroid malignant nodules is TIRADS 5, while the best threshold for C-TIRADS to diagnose thyroid malignant nodules is C-TIRADS 4C, other than CTIRADS 5, and the assessment results of C-TIRADS are different from guidelines, indicating that the threshold for malignant risk of C-TIRADS can occur deviation or variation, and the reason may be that some malignant nodules do not present sufficient sonographic features in very small size, and these led to inappropriate scoring and lower diagnostic performance. Similar condition has also occurred in other study, e.g., Basha et al found that in their multicenter prospective study on validity of ACR-TIRADS based 948 thyroid nodules, the best cut-off value for predicting malignant thyroid nodules was > ACR-TIRADS 3 , other than ACR-TIRADS 5 (Basha et al. 2018).

In this study, both C-TIRADS 4C and ACR-TIRADS 5 showed similar excellent specificity and NPV, indicating that they are better in differentiating malignant from benign thyroid nodules. This suggests that simplified scoring and categorization for thyroid nodule is benefit to patients and radiologists, and fine needle aspiration biopsy for lower malignant risk nodules may spare, for the thyroid cancer is indolent, it's not so urgent to practice biopsy, and follow-up may be an alternative.

The C-TIRADS and ACR-TIRADS presented comparable diagnostic performance in this study, however, unlike ACR-TIRADS has been validated by many studies, the C-TIRADS is a relatively recently launched system, and further validation is required in future (Basha et al. 2018; Ha et al.2018; Hoang et al.2018; Magri et al.2020; Middleton et al.2019; Peng et al.2020; Zhou et al.2020).

This study has some limitations: (1) This study is a retrospective design, although standardized examination and standardized image acquisition are used in the previous ultrasound examination, static image storage may affect the evaluation of various ultrasonic features, especially the determine of very hypoechoic, nodular margin and tiny liquid around the solid composition, and real-time dynamic display can evaluate each ultrasonic feature more accurately. (2) The number of nodules included in this study is not large enough, and surgery and pathology are regarded as the "gold standard", instead of the combination of surgery and FNA cytology, which cannot fully include benign lesions, which may inevitably induce selection bias. (3) In this study, the malignant nodules are mainly papillary carcinoma (96.88\%), while the benign nodules are nodular goiter $(93.32 \%)$, and the pathological types are relatively narrow. Therefore, in the future, a large sample should be studied to evaluate the diagnostic efficiency of medullary carcinoma, follicular carcinoma and Anaplastic carcinoma.

\section{Conclusions}

C-TIRADS and ACT-TIRADS have very good diagnostic performance in differentiating malignant from benign thyroid nodules by each optimized cut-off value, and the diagnostic performance of the fewer parameters based C-TIRADS 4C is comparable to the multiple parameters based ACR-TIRADS 5. The AUC of C-TIRADS $4 \mathrm{C}$ for the thyroid nodules with maximal diameter $<10 \mathrm{~mm}$ is significantly lower than the 
AUC of C-TIRADS 4C for thyroid nodules of all size, and is significantly lower than that of ACR-TIRADS 5 for the thyroid nodules with maximal diameter $<10 \mathrm{~mm}$. The diagnostic efficiency of C-TIRADS $4 \mathrm{C}$ for thyroid nodule with maximal diameter $<10 \mathrm{~mm}$ is poorer than that for larger size nodule.

\section{Declarations}

\section{Conflict of Interest Statement}

The authors declare they have no conflict of interests relating to this article.

\section{References}

Basha MAA, Alnaggar AA, Refaat R, El-Maghraby AM, Refaat MM, Abd Elhamed ME, Abdalla AAEM, Aly SA, Hanafy AS, Mohamed AEM, Afifi AHM, Harb O. The validity and reproducibility of the thyroid imaging reporting and data system (TI-RADS) in categorization of thyroid nodules: Multicentre prospective study. Eur J Radiol 2019; 117:184-92.

Cameselle-Teijeiro JM, Sobrinho-Simões M. New WHO classification of thyroid tumors: a pragmatic categorization of thyroid gland neoplasms. Endocrinol Diabetes Nutr 2018; 65:133-5.

Fradin JM. ACR TI-RADS: An advance in the management of thyroid nodules or Pandora's box of surveillance? J Clin Ultrasound 2020; 48:3-6.

Grani G, Lamartina L, Ascoli V, Bosco D, Biffoni M, Giacomelli L, Maranghi M, Falcone R, Ramundo V, Cantisani V, Filetti S, Durante C. Reducing the Number of Unnecessary Thyroid Biopsies While Improving Diagnostic Accuracy: Toward the "Right" TIRADS. J Clin Endocrinol Metab 2019; 104:95-102.

Jiang H, Tian Y, Yan W, Kong Y, Wang H, Wang A, Dou J, Liang P, Mu Y. The Prevalence of Thyroid Nodules and an Analysis of Related Lifestyle Factors in Beijing Communities. Int J Environ Res Public Health $2016 ; 13: 442$.

Ha EJ, Na DG, Baek JH, Sung JY, Kim JH, Kang SYUS. US Fine-Needle Aspiration Biopsy for Thyroid Malignancy: Diagnostic Performance of Seven Society Guidelines Applied to 2000 Thyroid Nodules. Radiology 2018; 287:893-900.

Ha EJ, Na DG, Moon WJ, Lee YH, Choi N. Diagnostic Performance of Ultrasound-Based Risk-Stratification Systems for Thyroid Nodules: Comparison of the 2015 American Thyroid Association Guidelines with the 2016 Korean Thyroid Association/Korean Society of Thyroid Radiology and 2017 American College of Radiology Guidelines. Thyroid 2018; 28:1532-7.

Ha SM, Baek JH, Choi YJ, Chung SR, Sung TY, Kim TY, Lee JH. Malignancy risk of initially benign thyroid nodules: validation with various Thyroid Imaging Reporting and Data System guidelines. Eur Radiol 2019; 29:133-40. 
Haugen BR, Alexander EK, Bible KC, Doherty GM, Mandel SJ, Nikiforov YE, Pacini F, Randolph GW, Sawka AM, Schlumberger M, Schuff KG, Sherman SI, Sosa JA, Steward DL, Tuttle RM, Wartofsky L. 2015 American Thyroid Association Management Guidelines for Adult Patients with Thyroid Nodules and Differentiated Thyroid Cancer: The American Thyroid Association Guidelines Task Force on Thyroid Nodules and Differentiated Thyroid Cancer. Thyroid 2016; 26:1-133.

Hoang JK, Middleton WD, Farjat AE, Langer JE, Reading CC, Teefey SA, Abinanti N, Boschini FJ, Bronner AJ, Dahiya N, Hertzberg BS, Newman JR, Scanga D, Vogler RC, Tessler FN. Reduction in Thyroid Nodule Biopsies and Improved Accuracy with American College of Radiology Thyroid Imaging Reporting and Data System. Radiology 2018; 287:185-93.

Horvath E, Majlis S, Rossi R, Franco C, Niedmann JP, Castro A, Dominguez M. An ultrasonogram reporting system for thyroid nodules stratifying cancer risk for clinical management. J Clin Endocrinol Metab 2009; 94:1748-51.

Kitahara CM, Sosa JA. The changing incidence of thyroid cancer. Nat Rev Endocrinol 2016; 12:646-53.

Kwak JY, Han KH, Yoon JH, Moon HJ, Son EJ, Park SH, Jung HK, Choi JS, Kim BM, Kim EK. Thyroid imaging reporting and data system for US features of nodules: a step in establishing better stratification of cancer risk. Radiology 2011; 260:892-9.

Magri F, Chytiris S, Croce L, Molteni M, Bendotti G, Gruosso G, Tata Ngnitejeu S, Agozzino M, Rotondi M, Chiovato L. Performance of the ACR TI-RADS and EU TI-RADS scoring systems in the diagnostic work-up of thyroid nodules in a real-life series using histology as reference standard. Eur J Endocrinol 2020; 183:521-8.

Middleton WD, Teefey SA, Reading CC, Langer JE, Beland MD, Szabunio MM, Desser TS. Multi institutional Analysis of Thyroid Nodule Risk Stratification Using the American College of Radiology Thyroid Imaging Reporting and Data System. AJR Am J Roentgenol 2017; 208:1331-41.

Moon JH, Hyun MK, Lee JY, Shim Jl, Kim TH, Choi HS, Ahn HY, Kim KW, Park DJ, Park YJ, Yi KH. Prevalence of thyroid nodules and their associated clinical parameters: a large-scale, multicenter-based health checkup study. Korean J Intern Med 2018; 33:753-62.

Morris LG, Tuttle RM, Davies L. Changing Trends in the Incidence of Thyroid Cancer in the United States. JAMA Otolaryngol Head Neck Surg 2016; 142:709-11.

Peng JY, Pan FS, Wang W, Wang Z, Shan QY, Lin JH, Luo J, Zheng YL, Hu HT, Ruan SM, Liang JY, Xie XY, Lu MD. Malignancy risk stratification and FNA recommendations for thyroid nodules: A comparison of ACR TI-RADS, AACE/ACE/AME and ATA guidelines. Am J Otolaryngol 2020; 41:102625.

Russ G, Bonnema SJ, Erdogan MF, Durante C, Ngu R, Leenhardt L. European Thyroid Association Guidelines for Ultrasound Malignancy Risk Stratification of Thyroid Nodules in Adults: The EU-TIRADS. 
Eur Thyroid J 2017; 6:225-37.

Shin JH, Baek JH, Chung J, Ha EJ, Kim JH, Lee YH, Lim HK, Moon WJ, Na DG, Park JS, Choi YJ, Hahn SY, Jeon SJ, Jung SL, Kim DW, Kim EK, Kwak JY, Lee CY, Lee HJ, Lee JH, Lee JH, Lee KH, Park SW, Sung JY; Korean Society of Thyroid Radiology (KSThR) and Korean Society of Radiology. Ultrasonography Diagnosis and Imaging-Based Management of Thyroid Nodules: Revised Korean Society of Thyroid Radiology Consensus Statement and Recommendations. Korean J Radiol 2016; 17:370-95.

Tessler FN, Middleton WD, Grant EG, Hoang JK, Berland LL, Teefey SA, Cronan JJ, Beland MD, Desser TS, Frates MC, Hammers LW, Hamper UM, Langer JE, Reading CC, Scoutt LM, Stavros AT. ACR Thyroid Imaging, Reporting and Data System (TI-RADS): White Paper of the ACR TI-RADS Committee. J Am Coll Radiol 2017; 14:587-95.

Xu T, Wu Y, Wu RX, Zhang YZ, Gu JY, Ye XH, Tang W, Xu SH, Liu C, Wu XH. Validation and comparison of three newly-released Thyroid Imaging Reporting and Data Systems for cancer risk determination.

Endocrine 2019; 64:299-307.

Yoon SJ, Na DG, Gwon HY, Paik W, Kim WJ, Song JS, Shim MS. Similarities and Differences Between Thyroid Imaging Reporting and Data Systems. AJR Am J Roentgenol 2019; 213: W76-W84.

Zhou J, Song Y, Zhan W, Wei X, Zhang S, Zhang R, Gu Y, Chen X, Shi L, Luo X, Yang L, Li Q, Bai B, Ye X, Zhai H, Zhang H, Jia X, Dong Y, Zhang J, Yang Z, Zhang H, Zheng Y, Xu W, Lai L, Yin L; Superficial Organ and Vascular Ultrasound Group of the Society of Ultrasound in Medicine of Chinese Medical Association; Chinese Artificial Intelligence Alliance for Thyroid and Breast Ultrasound. Thyroid imaging reporting and data system (TIRADS) for ultrasound features of nodules: multicentric retrospective study in China. Endocrine. 2020 Aug 27. doi: 10.1007/s12020-020-02442-x.

Zhou J, Yin L, Wei X, Zhang S, Song Y, Luo B, Li J, Qian L, Cui L, Chen W, Wen C, Peng Y, Chen Q, Lu M, Chen M, Wu R, Zhou W, Xue E, Li Y, Yang L, Mi C, Zhang R, Wu G, Du G, Huang D, Zhan W; Superficial Organ and Vascular Ultrasound Group of the Society of Ultrasound in Medicine of the Chinese Medical Association; Chinese Artificial Intelligence Alliance for Thyroid and Breast Ultrasound. 2020 Chinese guidelines for ultrasound malignancy risk stratification of thyroid nodules: the C-TIRADS. Endocrine 2020; 70:256-79.

\section{Figures}



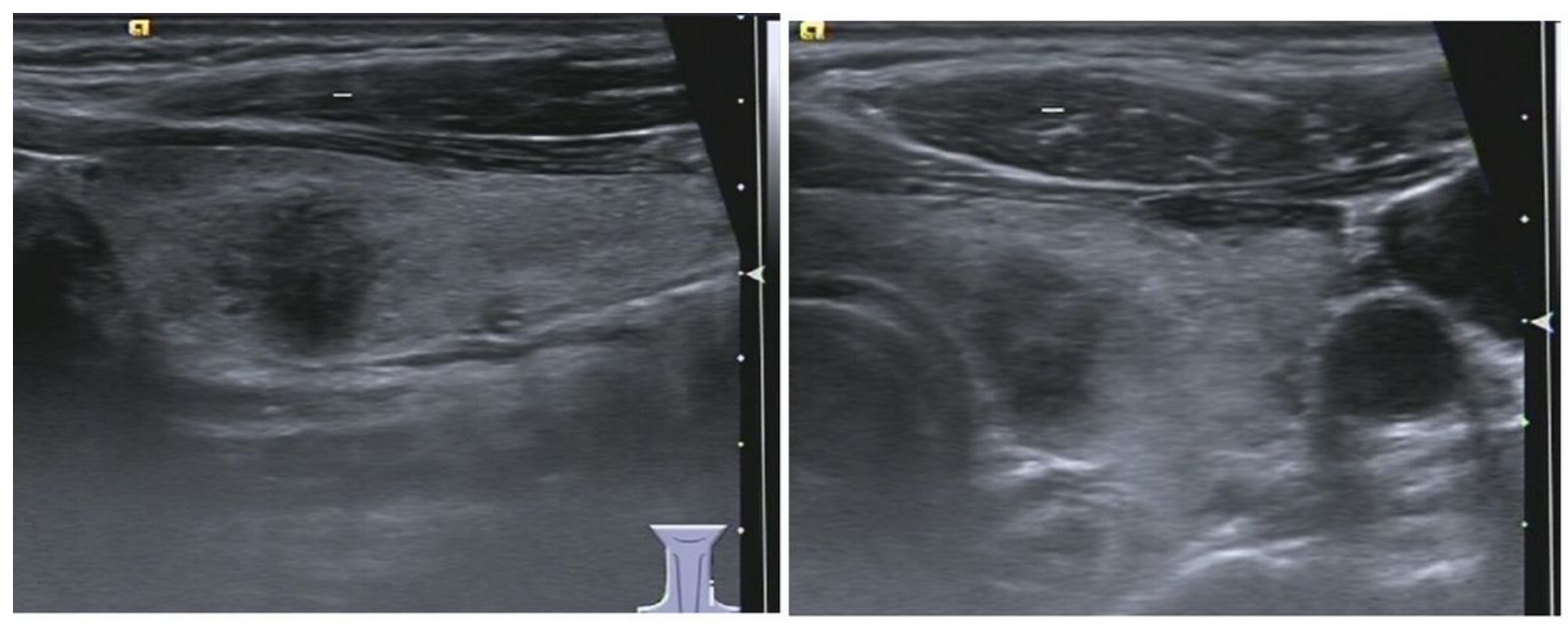

A

B

\section{Figure 1}

Figure 1a Longitudinal scanning sonography of a 37-year-old woman with thyroid nodule. Figure 1b Cross scanning sonography of a 37-year-old woman with thyroid nodule. The nodule locates at left lobe of the thyroid, characterized by $9.8 \mathrm{~mm} \otimes 9.2 \mathrm{~mm} \otimes 9.4 \mathrm{~mm}$ in size, solid composition, irregular shape, $A>T$ orientation, hypoechoic, ill-defined margin, and absence of posterior acoustic effect. C-TIRADS 4C, ACRTIRADS 5. Histopathology confirmed it is a chronic lymphocytic thyroiditis.

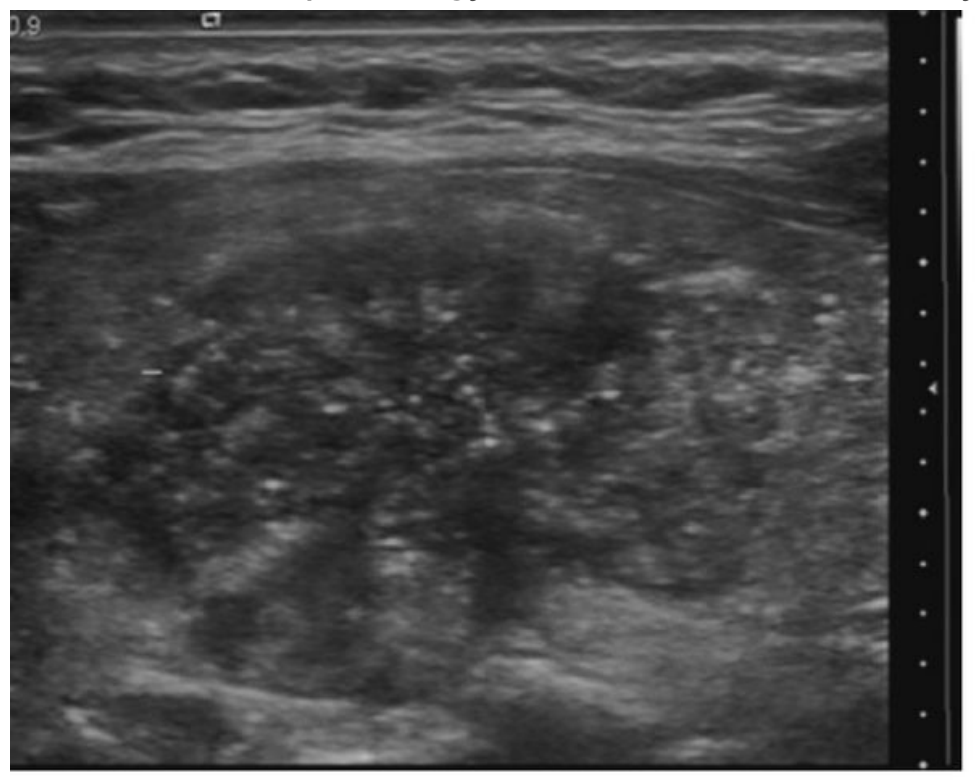

A

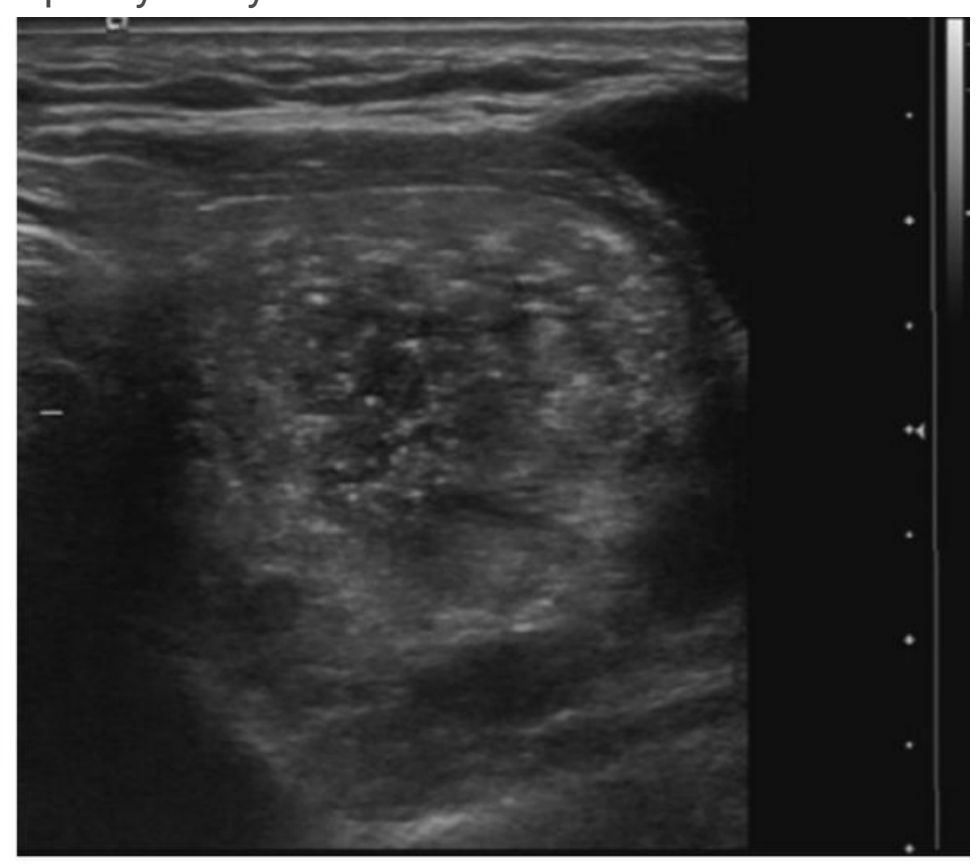




\section{Figure 2}

Figure 2a Longitudinal scanning sonography of a 45-year-old woman with thyroid nodule. Figure $2 \mathrm{~b}$ Cross scanning sonography of a 45-year-old woman with thyroid nodule. The nodule locates at left lobe of the thyroid, characterized by $21.2 \mathrm{~mm} \otimes 15 \mathrm{~mm} \otimes 13 \mathrm{~mm}$ in size, solid composition, irregular shape, $A<T$ orientation, heterogeneous iso/hypoechoic, numerous tiny punctate hyperechoic foci, ill-defined margin, and absence of posterior acoustic effect. C-TIRADS 4C, ACR-TIRADS 5 . Histopathology confirmed it is a papillary carcinoma.

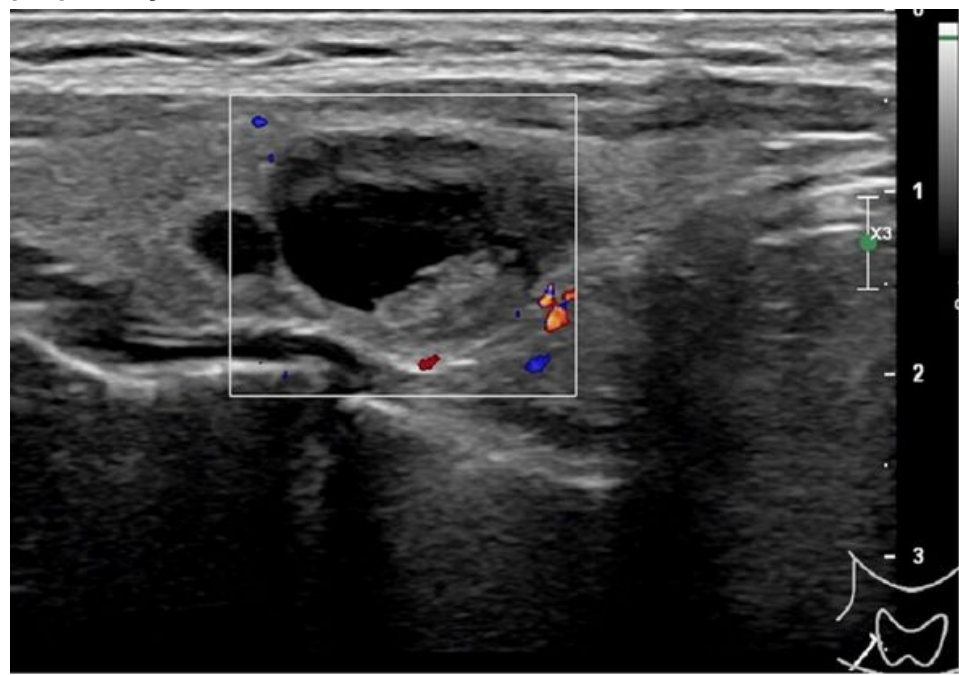

A

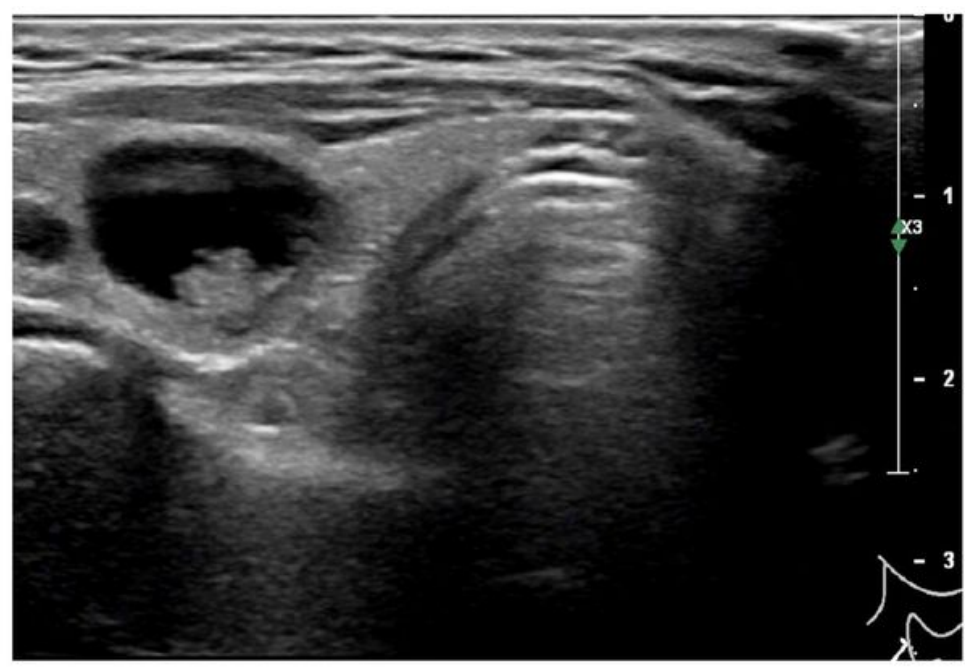

B

\section{Figure 3}

Figure 3a Longitudinal scanning sonography of a 53-year-old man with thyroid nodule. Figure $3 \mathrm{~b}$ Cross scanning sonography of a 53-year-old man with thyroid nodule. The nodule locates at right lobe of the thyroid, characterized by $20 \mathrm{~mm} \otimes 11.8 \mathrm{~mm} \otimes 10.7 \mathrm{~mm}$ in size, liquid and solid composition, irregular shape, $\mathrm{A}<\mathrm{T}$ orientation, dominantly anechoic and heterogeneous iso/hypoechoic, well-defined and discernable margin, and presence of posterior acoustic enhancement effect. C-TIRADS 3, ACR-TIRADS 3. Histopathology confirmed it is a papillary carcinoma. 